Instituto Internacional de Investigación y Desarrollo Tecnológico Educativo INDTEC, C.A.

DOI: https://doi.org/10.29394/Scientific.issn.2542-2987.2018.3.7.15.289-308

OAI-PMH: http://www.indteca.com/ojs/index.php/Revista Scientific/oai

\title{
Investigación Acción Participativa y Educación Ambiental
}

\author{
Autora: Yasmin Coromoto Requena Bolívar \\ Unidad Educativa Nacional de Talentos Deportivos del Estado Barinas, UENTADEBA
}

yasmile 2011@hotmail.com

Barinas, Venezuela

\section{Resumen}

La participación comprometida de los habitantes en diversas comunidades venezolanas es fundamental en la búsqueda de solución a problemas ambientales que enfrentan en la cotidianidad; ante esta realidad, se abordaron estudios fundamentados en Investigación Acción Participativa, a través de una revisión y análisis documental de cuatro trabajos relacionados con la participación comunitaria, realizados en el estado Lara. Para ello, se formuló la siguiente interrogante: ¿Cuál fue el logro en la solución de problemas ambientales en las comunidades, reportado a través de los trabajos de grado de maestría orientados bajo la investigación acción participativa y presentados ante la Universidad Yacambú en el lapso 2011-2013? Se emplea un enfoque cualitativo, abordando la información según las etapas sugeridas por Arias (2012): Búsqueda de fuentes, lectura inicial de documentos, elaboración del esquema preliminar, recolección de datos, análisis e interpretación de la información, formulación del esquema definitivo, introducción y conclusiones, informe final. Se inicia con la definición de las unidades de análisis e indagación de la literatura, a través de posturas teóricas, conceptos y aportes sobre: investigación acción participativa, participación y educación ambiental, para culminar con el análisis e interpretación de la información y las conclusiones de esta investigación. Para la recolección de los datos se utilizaron las fichas bibliográficas con la finalidad de organizar la información sobre las investigaciones consultadas, y de resumen para la síntesis de los documentos. Se concluyó que, en los trabajos de grado analizados, se cumplió con la finalidad de la IAP que consiste en la transformación de la situación-problema, lo que permitió que la IAP se convirtiera en el escenario propicio para promover la participación y la educación ambiental no formal.

Palabras clave: investigación participativa; participación comunitaria; educación ambiental. 


\section{Participant Action Research and Environmental Education}

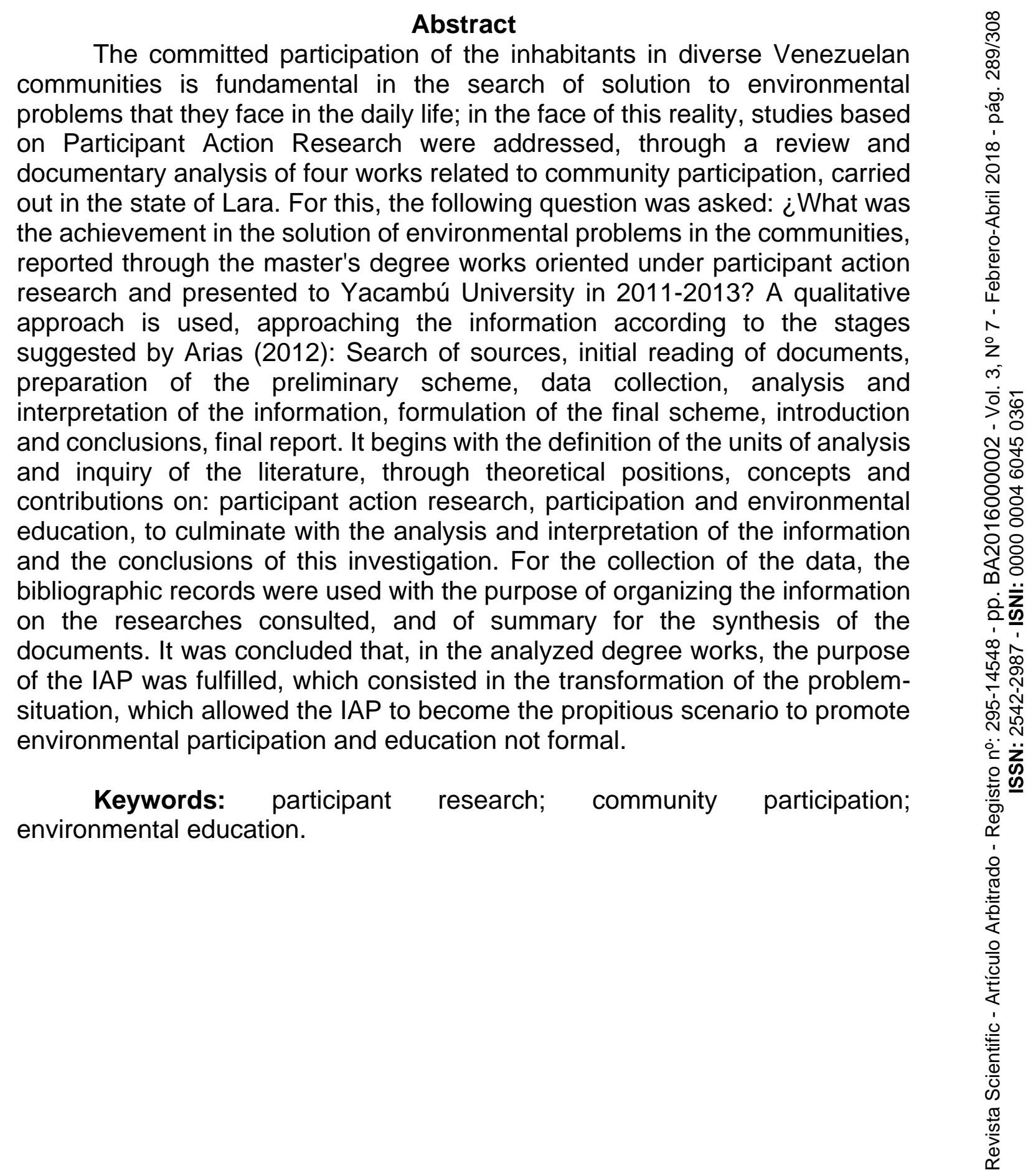

Date Received: 23-08-2017

Date Acceptance: 20-11-2017 


\section{Introducción}

En la actualidad, se pueden observar las evidencias de los cambios en el entorno natural debido a las acciones antrópicas, para la satisfacción de las necesidades del ser humano en pro de una mejor calidad de vida, pero afectando de manera alarmante, la interdependencia que debe existir entre él y la naturaleza, además de comprometer la permanencia del hombre en la Tierra. Esta afectación del medio se relaciona con el desmesurado desarrollo poblacional e industrial generado a través del tiempo, trasformando al ambiente de forma notable, conduciendo a un escenario crítico de agotamiento de los recursos naturales y contaminación en todos los ámbitos.

En este sentido, la educación ambiental adquiere la relevancia como eje de formación y sensibilización para la toma de conciencia, así como en el fomento de valores ecológicos, pero la puesta en práctica de diversas acciones en colectivo será el eje dinamizador para el cambio de actitud y la consolidación de los mencionados valores, que fomentarán un comportamiento proambiental consustanciado con el mejoramiento de las condiciones de vida en un entorno saludable y sustentable.

Se quiere con ello significar, que la formación ambiental desempeña un papel primordial en la conformación de una conciencia sobre el saber ecológico, puesto que a través de ella puede fluir el conocimiento necesario en la ciudadanía para fomentar el desarrollo de la sustentabilidad como tal, y una mayor sensibilización orientada a la conservación del patrimonio natural.

En este contexto, la investigación acción participativa es un método con el cual se puede propiciar la participación y la toma de conciencia hacia el mejoramiento de la calidad de vida, a través de la ejecución de acciones colectivas ambientalistas. Por ello, se presenta este trabajo que pretende mostrar los resultados de una investigación documental llevada a cabo, con la finalidad de realizar una revisión crítica de la experiencia que se presenta en cuatro investigaciones seleccionadas de manera intencionada. 
Las investigaciones fueron realizadas en el estado Lara (Venezuela), entre los años 2011 y 2013, con la particularidad que son trabajos de grado presentados ante la Universidad Yacambú de la ciudad de Barquisimeto (Lara), en el marco de la Maestría en Educación Ambiental. Es relevante señalar que, se describen los elementos conceptuales sobre la Investigación Acción, participación y educación ambiental, en lo que corresponde a la revisión de literatura; posteriormente se presenta la revisión de las investigaciones involucradas en el estudio; con el propósito de verificar si la IAP, en realidad, se convierte en un escenario propicio para la promoción de la educación ambiental en la solución de problemas; finalmente se incorporan las conclusiones que emergieron en la investigación documental.

\section{Revisión de la Literatura}

\subsection{Investigación Acción}

La investigación acción, es un término que acuñó y desarrolló Kurt Lewin en varias de sus investigaciones. Es un enfoque metodológico que se centra en el encuentro y contacto directo con las personas, para lograr un cambio en una determinada situación. Al respecto, Pérez (1998), afirma que se caracteriza por el procesamiento de datos y mejora de la realidad comprendida por cada uno de los entes involucrados, siendo flexible, democrática y abierta, además de emplear una variedad de elementos que contribuyen con la toma de decisiones.

En este sentido, todos los miembros participan en la mejora de la sociedad por medio de la investigación y se involucran en el aprendizaje permanente mediante una organización autogestora, con un mayor acercamiento a la realidad de estudio, lo que propicia la incorporación de todos como copartícipes en sus propias situaciones y la búsqueda de alternativas para mejorarla, basadas en la corresponsabilidad de todos, ante las diferentes situaciones que se presenten en su entorno. 
La Investigación Acción Participativa es la metodología de la intervención social, propia de la Psicología Social Comunitaria, tal como lo plantea Montenegro (2004): hace énfasis en que las personas afectadas por los problemas sociales deben ser parte de la solución de éstos, por tanto, el diseño, ejecución y evaluación de los planes y acciones, se hace a partir del diálogo entre los coinvestigadores y los miembros de la comunidad.

En concordancia con el planteamiento anterior, Balcázar (2003a), expone que la Investigación Acción Participativa (IAP) promueve la participación de los miembros de las diversas comunidades en la búsqueda de soluciones a sus propios problemas; además que provee un contexto concreto para involucrar a los actores en el proceso de investigación de una manera no tradicional como agentes de cambio.

\subsubsection{Fases de la Investigación Acción}

Para el proceso de investigación, Elliot (citado por Sandín, 2003a) propone las siguientes fases:

\subsubsection{Diagnóstico}

Para todo proceso es de suma importancia realizar la indagación de la situación a estudiar, a manera de tener conocimiento de la realidad e identificar qué se debe modificar. Se considera importante poseer conocimiento de la situación de la comunidad teniendo como objetivo la visión sistemática y holística del entorno; analizando las fortalezas y debilidades de la colectividad, y permite sistematizar de manera ordenada toda la información.

\subsubsection{Elaboración de un Plan de Acción}

Para desarrollar un plan se requiere de la organización, jerarquización de los problemas existentes, por los actores implicados, porque son ellos lo que deben seleccionar, proponer las posibles alternativas, y plantear de qué 
manera se van a lograr, así como los posibles actores responsables de cada actividad. Al respecto, Balcázar (2003b), opina que es aquí donde los participantes aprenden a desarrollar la observación, que les permite identificar las causas de sus problemas y las posibles soluciones.

\subsubsection{Ejecución del Plan de Acción}

En la fase anterior se logran establecer de manera colectiva, diferentes alternativas para la solución de la problemática existente, y guiadas por el objetivo de la investigación acción que implica la trasformación de la situación real; en esta fase, corresponde ejecutar cada una de las actividades propuestas, tomando en cuenta la flexibilidad. En este sentido, Balcázar (2003c), expone que los participantes llevan a cabo cada una de las actividades y acciones planificadas, con los recursos con que ellos cuentan y el apoyo de otros gremios, hasta llegar a solucionar cada uno de los problemas detectados en el diagnóstico.

\subsubsection{Evaluación e Interpretación del Plan de Acción}

En esta fase se lleva a cabo la reflexión sobre el plan de acción, y se describe cada una de las actividades desarrolladas de manera detallada, además de la revisión de los resultados obtenidos, al mismo tiempo se compara lo que se planificó con lo que en realidad se logró. Es importante que cada actividad al ser culminada sea evaluada por los coinvestigadores, con la finalidad de planificar los posibles correctivos que hagan falta.

Es importante acotar que, estas fases cumplen un ciclo de espiral sugerido por Elliot (citado por Sandín, 2003b), lo que permite la flexibilidad del proceso y la adaptación de cada una de las fases a las necesidades reales de la comunidad y en atención a las características de la situación, así como del logro de los objetivos previstos en el plan de acción. 


\subsection{Participación Comunitaria}

La participación comunitaria es un proceso cambiante y variable, lo cual está vinculado con las actitudes, toma de decisiones y relación entre los individuos, que en palabras de Bronfman y Gleizer (1994), se puede concebir como una forma para la solución de problemas de diversa índole, que es autogestionaria y brinda a la comunidad autosuficiencia e independencia, aumentando su nivel de autonomía.

En este sentido, la participación es considerada como una parte importante y fundamental para el desarrollo del ser humano; y un aspecto relevante es que está representada por la unión y cooperación de una comunidad en general. Según el Programa de las Naciones Unidas para el Desarrollo Humano (1993), la participación significa que "la gente intervenga estrechamente en los procesos económicos, sociales, culturales y políticos que afecten a sus vidas" (pág. 2).

De lo planteado anteriormente, se evidencia que la participación está estrechamente relacionada con el desarrollo del ser humano, puesto que parte desde su acción en todo su entorno y desenvolvimiento en la sociedad, con La relevancia de que genera bienestar a la comunidad en la búsqueda de soluciones a los problemas de diversa índole a través de la incorporación de sus habitantes.

En Venezuela la participación es considerada en la Constitución de la República Bolivariana de Venezuela (2009a), específicamente en el artículo 58 que la estipula como un derecho que tienen los sectores sociales, lo que implica la incorporación en la elaboración de propuestas, y en la identificación de prioridades. De este modo, es necesario que dentro de una comunidad los ciudadanos se sientan libres de crear, diseñar, ejecutar y evaluar acciones que se realicen de manera democrática para garantizar un desarrollo sustentable. Al respecto, Gabaldón (2006a), señala que sólo a partir de la libertad, los ciudadanos tienen la posibilidad de construir un tipo de desarrollo que 
presupone una amplia y transparente participación de la sociedad civil en las decisiones que la afectan.

\subsection{Educación Ambiental}

El escenario global se encuentra inmerso en conflictos ambientales, razón primordial para que se lleve a cabo una formación orientada hacia la generación de una conciencia conservacionista y un comportamiento en pro del mejoramiento de las condiciones para la vida y la preservación del entorno natural. En tal sentido, es relevante destacar a Gabaldón (2006b), quien opina que: "La identificación de los problemas ambientales y cómo controlarlos requiere un nuevo tipo de educación que a través de un juicio teórico y práctico del medio biótico y físico cree el interés y el amor por la naturaleza" (pág. 27).

De allí, la importancia de la puesta en práctica de la educación ambiental, que según la Ley Orgánica del Ambiente (2006), la define como un "proceso continuo, interactivo e integrador" que se logrea a través de la adquisición de conocimientos y experiencias por parte del ser humano, quien los comprende y analiza, los internaliza y los traduce en comportamientos favorables hacia el ambiente, fundamentado en valores ecológicos y actitudes proambientales que lo preparen para participar protagónicamente en la gestión del ambiente y el desarrollo sustentable.

Asimismo, la Constitución de la República Bolivariana de Venezuela (2009b), en el Artículo 107, establece que la educación ambiental es obligatoria en todos los niveles y modalidades del sistema educativo, haciendo referencia desde la perspectiva formal y no formal; incentivando la participación de las comunidades en la lucha por solucionar sus problemas.

Los objetivos de la educación ambiental fueron definidos en el Seminario Internacional de Educación Ambiental, por la Organización de las Naciones Unidas para la Educación, la Ciencia y la Cultura, Programa de las Naciones Unidas para el Medio Ambiente en 1975, estipulando un sistema de 
normas y pautas que debe incitar al ser humano a conocer todo lo relacionado con el mundo, saber cómo funciona, cómo son sus relaciones, así como incorporarse consciente y activamente en el proceso, en cuanto a la dinámica de la naturaleza y de la sociedad, a fin de actuar acorde con sus necesidades, y no simplemente retener información sobre aspectos precisos del ambiente.

\section{Método}

\subsection{Diseño de la Investigación}

La investigación se ubicó en un diseño documental que según Arias (2012), es "un proceso basado en la búsqueda, recuperación, análisis, crítica e interpretación de datos secundarios" (pág. 28). Cabe señalar que los datos secundarios son los obtenidos y registrados por otros investigadores en fuentes documentales: impresas, audiovisuales o electrónicas; en este caso, se trata de documentos escritos considerados como publicaciones no periódicas, como son investigaciones llevadas a cabo como requisito para optar al grado de Magíster en Educación Ambiental en la Universidad Yacambú, en el estado Lara, Venezuela y que fueron presentadas en el lapso 2011-2013, bajo el enfoque de la Investigación Acción Participativa.

\subsection{Búsqueda de Fuentes}

Para el desarrollo de esta fase se plantearon criterios que permitieron ubicar las fuentes de manera confiable para la selección; estos criterios fueron: (a) Trabajos de grado de Maestría en Educación Ambiental presentados ante la Universidad Yacambú en el lapso 2011-2013; (b) Orientados bajo el método de Investigación Acción Participativa y (c) Dirigidos a la participación comunitaria en la solución de problemas y fundamentados en la educación ambiental.

En la revisión de fuentes se comprobó que se presentaron 67 trabajos de grado en el área de Educación Ambiental ante la Universidad Yacambú 
durante el lapso 2011-2013, de los cuales siete se orientaron bajo la investigación acción y de éstos solamente cuatro estuvieron dirigidos a la participación comunitaria en la solución de problemas: Hernández (2011a), García (2012a), Borges (2012a) y Goyo (2013a). Cabe destacar que los datos bibliográficos de estas investigaciones fueron recogidas a través de fichas para su respectiva clasificación.

\subsection{Análisis e Interpretación de la Información}

En atención a los criterios de selección, se procedió a realizar el análisis documental de las investigaciones mencionadas en el apartado anterior, tomando en cuenta como categorías a la Investigación Acción Participativa con la revisión de las respectivas fases que la componen; la participación comunitaria y la educación ambiental no formal, para lo cual se elaboró una matriz de análisis que permitió la posterior interpretación de la información.

Se inicia con el trabajo de Hernández (2011b), titulado "Acciones participativas ambientalistas orientadas hacia el mejoramiento de la calidad de vida de los habitantes del caserío Palo Verde en el municipio Andrés Eloy Blanco" específicamente en el estado Lara, donde existían situaciones que atentaban contra la calidad del ambiente y, por ende, de la calidad de vida de sus pobladores, motivando así el desarrollo de la investigación.

Entre las situaciones encontradas por Hernández (2001c), se tenía la dispersión de residuos sólidos por la comunidad; quema de basura, práctica inadecuada común en los patios de las casas; ausencia de mantenimiento de los tanques de almacenamiento de agua proveniente de las nacientes en la zona alta; problemas con la red de aguas servidas, que colapsaban y algunos pobladores dejaban que corrieran libremente por las calles; uso de biocidas en los terrenos productivos aledaños a la comunidad, así como la tala y quema.

Cabe señalar que la investigadora, logró elaborar con la participación de los actores sociales un plan de acción que contemplaba actividades de 
formación y de acción; entre las actividades de formación, desarrolló CharlasTaller, sobre "Disposición de residuos sólidos, clasificación, reutilización y reciclaje; "Participación comunitaria: Eje integrador para el mejoramiento de la calidad de vida" y "Uso racional del agua". Asimismo, llevó a cabo varias acciones con la participación de los pobladores, tales como: Operación limpieza de la comunidad, de las nacientes de agua y la toma de agua; jornada de reforestación; murales ecológicos; campaña informativa sobre el dengue; elaboración del proyecto sobre el agua; elaboración de papeleras ecológicas.

Es importante destacar los logros de Hernández (2011d), a través de la Investigación Acción Participativa, aplicando la observación participante, entrevistas en profundidad, grupos focales y asambleas comunitarias, que llevaron a desarrollar actividades orientadas por el plan de acción elaborado en el caserío Palo Verde, con la participación de los habitantes y el apoyo de seis coinvestigadores miembros del sector:

1. Se logró la sensibilización de los habitantes de la comunidad a través de las reflexiones generadas durante las reuniones de planificación y las charlas.

2. Se alcanzó la incorporación y participación de los habitantes en la solución de los problemas ambientales encontrados durante el diagnóstico.

3. Se redujeron los casos de dengue y enfermedades digestivas como las diarreas, gracias a la sensibilización de los habitantes y toma de conciencia, respecto a la eliminación de los criaderos de zancudos.

4. Se mantiene de manera permanente la participación de los pobladores en acciones orientadas a la conservación de los espacios naturales y el mantenimiento de las diferentes áreas.

En consecuencia, se puede expresar que en el caserío se generó un proceso de transformación social representado por la motivación a participar 
en las acciones orientadas al beneficio en común, contrario a la actitud apática y desidia encontrada al inicio de la investigación; además, la participación es activa y comprometida, sin la búsqueda de intereses particulares, sino más bien desinteresada y cooperativa.

Por otra parte, García (2012b), realizó su investigación titulada: "Integración escuela y comunidad en el barrio San Isidro de Sanare: Una experiencia de conservación ambiental" en la jurisdicción del municipio Andrés Eloy Blanco del estado Lara. La investigadora pudo observar que además de la situación que generaba el mercado itinerante al dejar una inmensurable cantidad de residuos sólidos; existían grandes cúmulos de éstos en las calles del barrio, aunado a la quema de basura.

Asimismo, la contaminación visual, originada por grafitis de todo tipo, aunado a focos de contaminación sónica originada por vehículos con equipos musicales a alto volumen a cualquier hora de la noche, y por último el uso inadecuado de biocidas en las parcelas aledañas. La situación motivó a la investigadora a desarrollar un proceso de investigación acción participativa que propiciara la integración de la comunidad con el Liceo Bolivariano Sanare, con sede en el barrio. Entre las actividades de formación se desarrollaron Charlas-Taller sobre "Educación ambiental"; "Legislación y factores de agresión ambiental"; "Uso racional del agua; "Uso de biocidas" y "Manejo de residuos sólidos".

En cuanto a las acciones llevadas a cabo con la participación conjunta de la institución educativa involucrada y de varios habitantes del barrio San Isidro, con apoyo de seis pobladores del sector que fungieron como coinvestigadores, se tienen las siguientes: Rally ecológico; elaboración de carteleras y papeleras; concurso literario; murales ecológicos y carnavales ecológicos. Los logros de estas actividades con la incorporación de los habitantes del barrio se pueden expresar en resumen así: 
1. A través de la reflexión que se generó por parte de los actores sociales involucrados en la investigación, en las diferentes charlas-talleres.

2. A partir de la ejecución de actividades, los habitantes se incorporaron hasta lograr la participación de varios vecinos de manera comprometida.

3. Los trabajadores del mercado itinerante, luego del rally ecológico y de asistir al taller sobre legislación ambiental, mostraron un cambio de actitud y al momento de retirarse, han recogido los residuos sólidos generados.

4. La imagen del barrio cambió y ahora es agradable a la vista de propios y visitantes, debido a la sustitución los grafitis sin sentido por murales ecológicos.

5. La participación de los actores en las actividades planificadas por la institución, así como de actividades por parte del consejo comunal orientadas hacia la conservación, es uno de los logros relevantes.

Cuando se revisan los logros de los objetivos del plan de acción, se evidencia que, a través de la ejecución de actividades, en el barrio San Isidro, se reconstruyó de manera colectiva el significado de la participación comunitaria, orientada a lograr la integración entre el Liceo Bolivariano Sanare y la comunidad, fundamentada a generar procesos de conservación ambiental.

En el mismo contexto larense, Borges (2012b), llevó a cabo una investigación titulada "Unidad Educativa Nacional Aguedo Felipe Alvarado de Bobare, como centro de quehacer comunitario en el marco del desarrollo endógeno sustentable" en el municipio Iribarren. La investigadora observó una problemática compleja para el cumplimiento de actividades orientadas al desarrollo endógeno, donde pudiesen incorporarse los habitantes de la comunidad, debido al poco o nulo intercambio de saberes entre la institución y la comunidad, poca voluntad hacia el cambio y la transformación social, inexistencia de incentivo hacia las innovaciones para el avance curricular, poca presencia de dinámicas vivenciales en las actividades académicas de aula; 
aunado a manifestaciones de conformismo, egoísmo, pesimismo e individualismo.

Ante esta realidad, se propuso llevar a cabo un proceso de investigación acción participativa involucrando a la institución y la comunidad, logrando desarrollar un plan de acción estructurado por los miembros de la comunidad educativa y la comunidad local, conformado por cuatro pilares fundamentales: sensibilización en liderazgo comunitario (divulgación informativa, campaña radial y carteleras); taller de liderazgo comunitario (escuela como centro de quehacer comunitario), expoferia artesanal y agropecuaria; y festival cultural, deportivo y recreativo comunitario (concurso literario, juegos tradicionales y voz estudiantil). Los logros de este plan de acción se manifiestan a través de:

1. Se impulsó el abordaje de la institución educativa como centro de saberes y quehaceres comunitarios; además de una amplia apertura de espacios para el análisis crítico reflexivo con docentes, estudiantes, representantes y líderes comunitarios, para asumir los principios del desarrollo endógeno sustentable y la escuela comunitaria.

2. Se promovió el acercamiento entre los sectores productivos locales y la institución, en el marco del desarrollo endógeno sustentable, con la participación de representantes artesanales y cría de caprinos, actividades fundamentales para el desarrollo endógeno sustentable de la parroquia Aguedo Felipe Alvarado.

Siendo el desarrollo endógeno un aspecto relevante en lo que compete a la conservación ambiental, los logros alcanzados en la investigación realizada por Borges (2012c), son una expresión de la importancia de la Investigación Acción Participativa para la consolidación de acciones colectivas con participación de la escuela y la comunidad en la solución de problemas y el acercamiento a conductas orientadas hacia la sustentabilidad, que 
propiciarán otros espacios comunitarios para el mejoramiento de la calidad de vida.

Por último, se tiene la investigación llevada a cabo por Goyo (2013b), titulada "Educación ambiental como eje integrador comunitario en el mejoramiento de las condiciones ambientales de las comunidades Los Jabillos y Los Mangos, municipio Palavecino". Este estudio surge de la inquietud del investigador por coadyuvar a los habitantes de los sectores mencionados a solucionar los problemas que enfrentaban, luego de una invitación que le realizar la Universidad Yacambú a participar en una actividad de apoyo comunitario.

En las visitas realizadas pudo corroborar situaciones como la acumulación de residuos sólidos en las diferentes calles y viviendas; restos de chatarras, maleza y basura en los patios y frentes de las casas; presencia de un botadero clandestino generado por una empresa cercana al sector; quema de basura, como una opción de ambas comunidades para desaparecer los cúmulos de residuos sólidos; derrame de aguas servidas a las calles de las comunidades, aunado a la presencia de muchos perros callejeros.

Ante esta situación y de manera conjunta con los habitantes de los sectores formuló un plan de acción estructurado por charlas sobre: "Manejo de residuos sólidos", "El dengue", "Quema de basura y los riesgos para la salud", "Legislación ambiental". Además de elaboración de murales ecológicos; rescate de la vía principal con jornadas de limpieza, desmalezado, pintura de frentes de las viviendas; actividades deportivas y culturales. Estas actividades contaron con la participación numerosa de los miembros de ambas comunidades compartiendo responsabilidades en los espacios comunes, como la vía principal, manifestándose los siguientes logros:

1. Incorporación comprometida de los habitantes de ambas comunidades en la solución de sus problemas ambientales comunes y 
particulares.

2. Cambio de actitud de los habitantes orientada hacia la reutilización de residuos sólidos, así como el contacto con empresas para el reciclaje de productos como plástico, aluminio, otros metales, papel y cartón, como proceso de autogestión para la casa comunal.

3. Cambio de apariencia de las comunidades, al mantener las calles libres de basura y las paredes con murales ecológicos, además de la reforestación de la calle principal y otras áreas del sector, así como por la ausencia de residuos sólidos en las calles.

4. Eliminación del derrame de aguas servidas, incorporando a la red de cloacas las viviendas que faltaban, a través de un trabajo comunal conjunto con la empresa hidrológica.

5. Proyección de las actividades a comunidades aledañas en vista de los logros obtenidos y en procura de los mismos objetivos, debido a la similitud de los problemas ambientales.

Estos logros son una evidencia de los resultados de llevar a cabo un proceso de investigación acción participativa para la solución de problemas ambientales, y como se incentiva en otras comunidades la participación, organización, solidaridad, cooperación, colaboración y trabajo en equipo para mejorar las condiciones de vida y asumir un comportamiento ambiental dirigido a la conservación del entorno.

\section{Conclusiones}

Luego de realizar una revisión crítica a las investigaciones que se presentan en este artículo, se tiene que de la misma se derivan varios aspectos relevantes que le otorgan la significatividad a la Investigación Acción Participativa como un escenario adecuado para la promoción de la educación ambiental, a través de las actividades de formación que se desarrollan en las 
comunidades para ofrecer información importante que permiten la gestión del conocimiento ambiental entre los miembros de las poblaciones que son intervenidas con la IAP; por otra parte, la mencionada formación genera la toma de conciencia necesaria para la participación activa y comprometida en las actividades planificadas en colectivo y orientadas a la solución de los problemas ambientales.

Lo descrito anteriormente, comprueba lo que expone Vio (1998), respecto a que la investigación acción participativa es un enfoque de investigación social que busca la plena participación de los actores involucrados en el análisis de su propia realidad, con el objeto de promover la transformación social para el beneficio de los participantes en la investigación. Por otra parte, es relevante destacar que en las investigaciones realizadas por Hernández (2011e), García (2012c), Borges (2012d) y Goyo (2013c), se ponen de manifiesto varios rasgos de la IAP, entre ellos que: el objeto de estudio se decide a partir de lo que interesa a un grupo, lo cual significa que esta metodología se aplica a situaciones de la vida real.

De igual manera, se cumplió con la finalidad de la IAP que consiste en la transformación de la situación-problema que afecta a la gente involucrada, siendo las personas los principales agentes de cambio y para lograrlo se apoya en actividades de formación, que en los casos que se revisaron correspondía a aspectos comprendidos en la dimensión ambiental. Por último, en las investigaciones estudiadas se comprende que el aporte de los investigadores correspondió en los aspectos teórico y metodológico, mientras que los actores involucrados contribuyeron con sus experiencias y conocimientos populares, siendo esta conjugación lo que permitió que la IAP se convirtiera en el escenario propicio para promover la participación y la educación ambiental no formal. 


\section{Referencias}

Arias, F. (2012). El proyecto de investigación: Introducción a la metodología científica. (6ta ed.) Venezuela: Episteme.

Balcázar, F. (2003a,b,c). Investigación Acción Participativa (IAP):

Aspectos conceptuales y dificultades de implementación.

Fundamentos en Humanidades. Año IV - N I/II (7). 2003, págs. 59-77.

Estados Unidos: Universidad Nacional de San Luis.

Borges, Y. (2013a,b,c,d). Unidad Educativa Nacional Aguedo Felipe Alvarado de Bobare, como centro de quehacer comunitario en el marco del desarrollo endógeno sustentable. Trabajo de Grado de Maestría no publicado. Barquisimeto, Venezuela: Universidad Yacambú.

Bronfman, M. \& Gleizer, M. (1994). Participación comunitaria: ¿Necesidad, excusa o estrategia? 0 de qué hablamos cuando hablamos de participación comunitaria. En: Cad. Saúde Públ., Rio de Janeiro, 10(1): 111-122, enero-marzo, 1994.

Constitución de la República Bolivariana de Venezuela (2009a,b). Gaceta

Oficial de la República Bolivariana de Venezuela, 5.908 (Extraordinario). Febrero 19, 2009.

Gabaldón, A. (2006a,b). Desarrollo sustentable: La salida de América Latina. Caracas, Venezuela: Grijalbo.

García, M. (2012a,b,c). Integración escuela y comunidad en el barrio San Isidro de Sanare: Una experiencia de conservación ambiental. Trabajo de Grado de Maestría no publicado. Barquisimeto, Venezuela: Universidad Yacambú.

Goyo, A. (2013a,b,c). Educación ambiental como eje integrador comunitario en el mejoramiento de las condiciones ambientales de las comunidades Los Jabillos y Los Mangos, municipio Palavecino. Trabajo de Grado de Maestría no publicado. Barquisimeto, 
Venezuela: Universidad Yacambú.

Hernández, Y. (2011a,b,c,d,e). Acciones participativas ambientalistas orientadas hacia el mejoramiento de la calidad de vida de los habitantes del caserío Palo Verde en el municipio Andrés Eloy

Blanco. Trabajo de Grado de Maestría no publicado. Barquisimeto, Venezuela: Universidad Yacambú.

Ley Orgánica del Ambiente (2006). Gaceta Oficial de la República Bolivariana de Venezuela, 5.833 (Extraordinario). Diciembre 22, 2006. Montenegro, M. (2004). La investigación acción participativa. En: Introducción a la Psicología Comunitaria. Barcelona, España: UCO.

Organización de las Naciones Unidas para la Educación, la Ciencia y la Cultura, Programa de las Naciones Unidas para el Medio Ambiente (1975). Carta de Belgrado. Un Marco General para la Educación Ambiental. Seminario Internacional de Educación Ambiental, 13-22 de octubre. [transcripción en línea]. Recuperado de:

http://unesdoc.unesco.org/images/0001/000177/017772sb.pdf

Pérez, G. (1994). Investigación cualitativa: Retos e interrogantes I. Métodos. Madrid: La Muralla.

Programa de las Naciones Unidas para el Desarrollo Humano (1993). Informe sobre Desarrollo Humano. [Documento en línea]. Recuperado de: http://hdr.undp.org/en/media/hdr 1993 es cap2.pdf

Sandín, M. (2003a,b). Investigación cualitativa en educación: Fundamentos y tradiciones. España: McGraw-Hill.

Vio, F. (1998). Investigación participativa y praxis rural. Santiago de Chile: CEOAL. 


\section{Yasmin Coromoto Requena Bolívar \\ e-mail: yasmile 2011@hotmail.com}

Nacida en Venezuela. Profesora especialista en

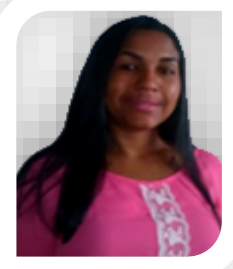

Biología (Universidad Pedagógica Experimental Libertador IMPM Barinas). Estudiante de postgrado en la Universidad Pedagógica Experimental Libertador IMPM Barinas. Programa: Educación Ambiente y Desarrollo. Docente de aula en Unidad Educativa Nacional de Talentos Deportivos del Estado Barinas. 